\title{
Validated Modelica Building Package for Energy Performance Simulation for Educational and Teaching Purposes
}

\author{
Shan Hua ${ }^{1}$ Fabian Reu $^{2} \quad$ Manuel Lindauer ${ }^{1} \quad$ Jochen Stopper $^{1}$ Christoph van Treeck ${ }^{3}$ \\ ${ }^{1}$ Centre for Sustainable Building, TU München, Germany, \{shan.hua, manuel.lindauer, \\ jochen. stopper\} atum. de \\ ${ }^{2}$ Institute of Energy Efficient and Sustainable Design and Building, TU München, Germany, \\ fabian.reuss@tum.de \\ ${ }^{3}$ Chair of Energy Efficiency and Sustainable Building E3D, RWTH Aachen University, Germany, \\ treeck@e3d.rwth-aachen.de
}

\begin{abstract}
This paper introduces the work and application of the Modelica IBPSBuilding package which has been developed at TU München. The aim of the package is to simulate building energy performance, especially the thermal behaviour of buildings. Its application is focused on research and education in order to help applicationoriented researchers and students to understand the physical processes of a building performance simulation and gain persuassive simulation results. The package describes all basic processes including conduction, convection, radiation and ventilation, and it is validated with German VDI-Guidelines.

Keywords: building modeling, energy simulation, adaptive façade
\end{abstract}

\section{Introduction}

The teaching module "Building Performance Modeling and Simulation" was established at TU München in 2006 which is based on a formerly created block course about simulation modeling using Maple. It consists of a lecture with the same name as the module and the practical exercise "Implementing a Building Performance Simulation". The lecture explains the physical basics of thermal building simulation including heat conduction, convection, short- and longwave radiation processes, but also introduces mathematical and numerical aspects of simulation.

Theories explained in the lectures are applied in the exercise, in which Modelica is used to create models of all the involved processes (van Treeck, 2010). These models evolved over the years and new ideas with a whole building model that had been developed in master theses and exercises were implemented. The development was conducted with OpenModelica since 2012 and then several functional enhancements and adaptations to research projects were conducted with ITI SimulationX. The actual state of the model package and the relative development is shown in this paper. Additionally, the methodology and results of a validation of the model package according the a German technical guideline is presented.

\section{The Library IBPSBuilding}

The aim of the IBPSBuilding library is to have a thermal simulation tool, in which every single process related to building thermal performance is fully understood and can be modified in any desired way in order to have maximal flexibility. Especially for the aspect of understanding every detail, it is necessary to create a library from scratch and not to use existing libraries.

The main structure (in Figure 1) of this model library meets the lecture syllabus, and contents are complemented and explained to students step by step along the pace of the lecture. Taking advantage of the polymorphism in Modelica language, complicated physical processes and building elements are designed to be based on abstract concepts and structures. Base classes for interfaces, functions and records for materials are arranged into sub-packages, which are provided as modules that students can use to create their own elements for heat transfer processes and construction elements. The methodology of development of the standard components of heat transfer processes provided in this library is introduced in section 2.1. At the end of each semester, typical rooms should have been created and simulated by the students with support of the lecturers. The simulation results of these test cases can be used to get insights on the most responsive proccesses and indicators of thermal building performance.

In order to let the library structure be commensurate to the domain structure of a BIM data format like IFC, a skeletal framework is provided by a master thesis for de- 


\begin{tabular}{|c|c|}
\hline \multicolumn{2}{|c|}{ P IBPSBuilding } \\
\hline$\pm \mathbf{P}$ & Constructions \\
\hline$\pm \mathbf{P}$ & HeatTransferUnits \\
\hline$\pm \mathbf{P}$ & Interfaces \\
\hline$\pm \mathbf{P}$ & MaterialLibrary \\
\hline$\pm \mathbf{P}$ & AmbientConditions \\
\hline$\pm \mathbf{P}$ & Functions \\
\hline$\pm \mathbf{P}$ & Test \\
\hline
\end{tabular}

Figure 1. main structure of IBPSBuilding-package

velopment of appropriate interfaces in research according to this library (Hua, 2014).

\subsection{Implementation of heat transfer pro- cesses}

The heat transfer processes described in this library can be classified into two groups: the heat transfer processes determined by temperature difference, and the shortwave radiation unrelated to temperature. All the building elements are simplified into one-node thermal mass with capacity models of the material and the key positions, e.g. surfaces. Each building element is integrated into a chain of connections of various physical processes (Figure 2). Coefficients and properties for concretization of those physical processes are given as parameters or calculated dynamically in the construction component model.

In order to reduce the complexity of modeling and to increase the simulation efficiency on the building level, several restrictions are contained in the development and modeling:

1. Currently, thermo fluid processes are not considered in the teaching version. For any adoption with fluid materials, simulations are conducted under ideal conditions with a single heat capacity, convection and steady state mass transfer - pressure losses, change of density and enthalpy are disregarded in the current phase of development. So no interaction with elements in the 'Modelica.Fluid' library is adopted.

2. The discretization follows a nodal approach for calculating heat transfer through the building fabric in a one-dimensional manner, i.e. where heat transfer perpendicular to the main direction of heat flow is neglected. Multi-dimensional effects such as cold bridges are accordingly disregarded.

\subsubsection{Conduction and convection}

The modules for conduction and convection process are based on the same abstract partial model:

$$
\dot{Q}=C \cdot\left(T_{i}-T_{e}\right)
$$

Basic properties like the temperature difference between the two sides of a layer, the inlet / outlet heat flow etc. are declared as prescribed in the abstract model, so that programming effort can be minimized, especially if the concretized modules would be coded by students in practical fields, who are less familiar with programming. The coefficient $C$ is first specialized in different successors from the abstract model, e.g. in a conduction module for half of a solid material layer, $C=A \star 1$ ambda $/ d / 2$, here $A$ is the net area of a material layer, lamda is the material conductance, and $\mathrm{d}$ is the layer thickness.

Concretization of values of heat transfer coefficients for convection depends on the boundary condition of the building element very much. Within the concept of flexibility and full controllability, a bunch of parameters are added, so that it can be chosen if fixed values for the heat transfer coefficients should be assigned for validation or conceptual design phases, or the values should be calculated dynamically and internally according to geometry, wind velocity, temperature differences etc. A theoretical reference of most of the physical processes described in this package is from Clarke (2001). Chances are given to students who are taking part into the seminar to create new functions and codes to implement other theoretical methods. An example is to calculate the convection coefficients according to boundary conditions and tilt angle. A function calconvectioncoeff is implemented like this:

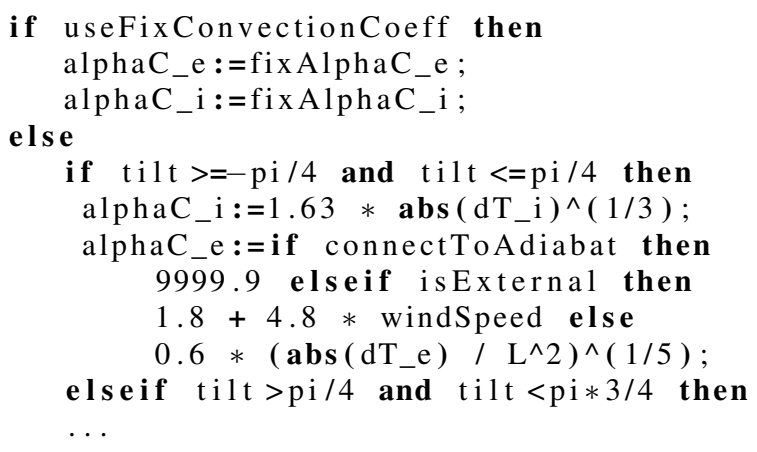

where the values of Boolean parameters such as useFixConvectioncoeff, connectToAdiabat and isExternal are assigned by high-level modelers from input-windows. The variable tilt is transmitted from the geometric parameter-set of the building element, and serves as the arbiter of which formula should be taken into consideration. 


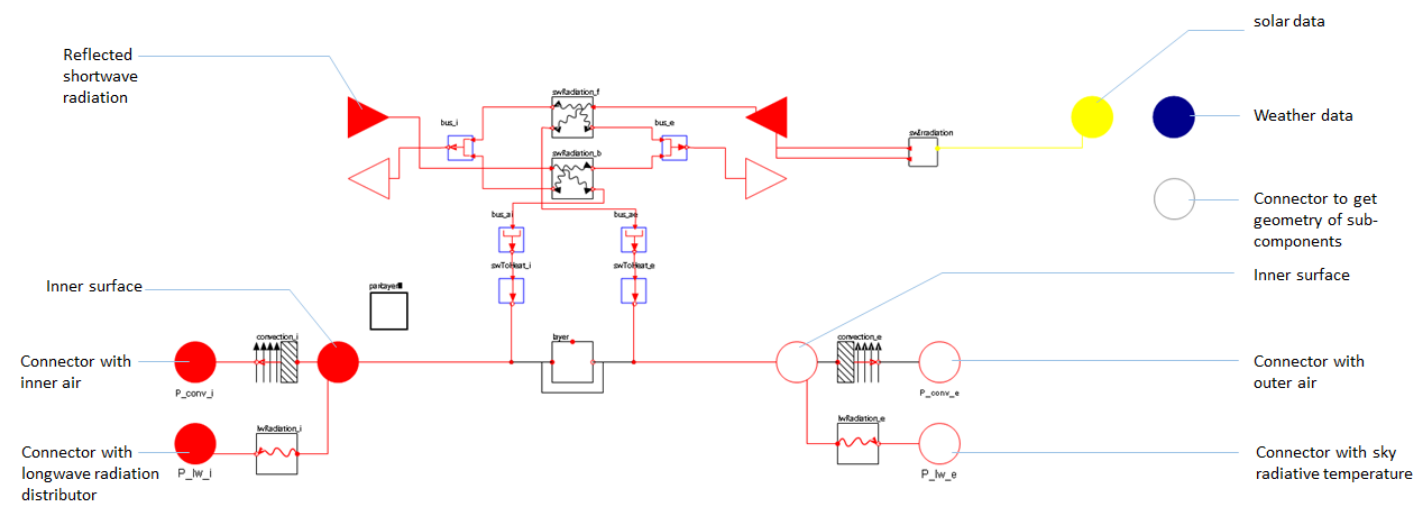

Figure 2. An opaque building element and its accessible connectors

\subsubsection{Radiation and window modeling}

By contrast with heat transfer models like conduction and convection, a shortwave radiation process is not based on HeatPorts ${ }^{1}$ with temperature as the potential variable because of its independence of the temperature difference, therefore the direction of the heat flow by radiation is given explicitly. Besides the heat flow variable, a factor is stored in a connector SwPort for distributions of radiation to its parent model. A shortwave radiation module has four SwPorts: one as input to receive incoming radiation, and the other three stand for the outputs through transmission, reflection and absorption processes. Calculation methods of the factors are first assigned either in inheritance or in instantiation process.

A transparent construction element should have four shortwave radiation modules because shortwave radiation takes place on a plane between two mediums (in case of window are glass and gas). Moreover, each glass pane includes two surfaces, and each of them conducts radiation from both sides, that is to say, from glass to gas, and from gas to glass. Intermediate connections are shown in the following figure 3 .

Within this structure, radiation inside a glass plane between the two surfaces are taken into account in numerical handling process by simulation solver. It can be solved less efficiently than an analytical method, but more clearly represented for non-programmers to help them understand actual physics behind the model. Absorbed energy is summed up into a hub, and converted into a thematic adaptable format into HeatPort, which can be connected to the layer heat capacitor. (connected with P_a, Figure 2)

\footnotetext{
${ }^{1}$ The connector HeatPort is inherited from the Modelica standard library - Modelica. Heat Transfer. Interfaces. It consists of a potential variable temperature and a flow variable heat flow rate.
}

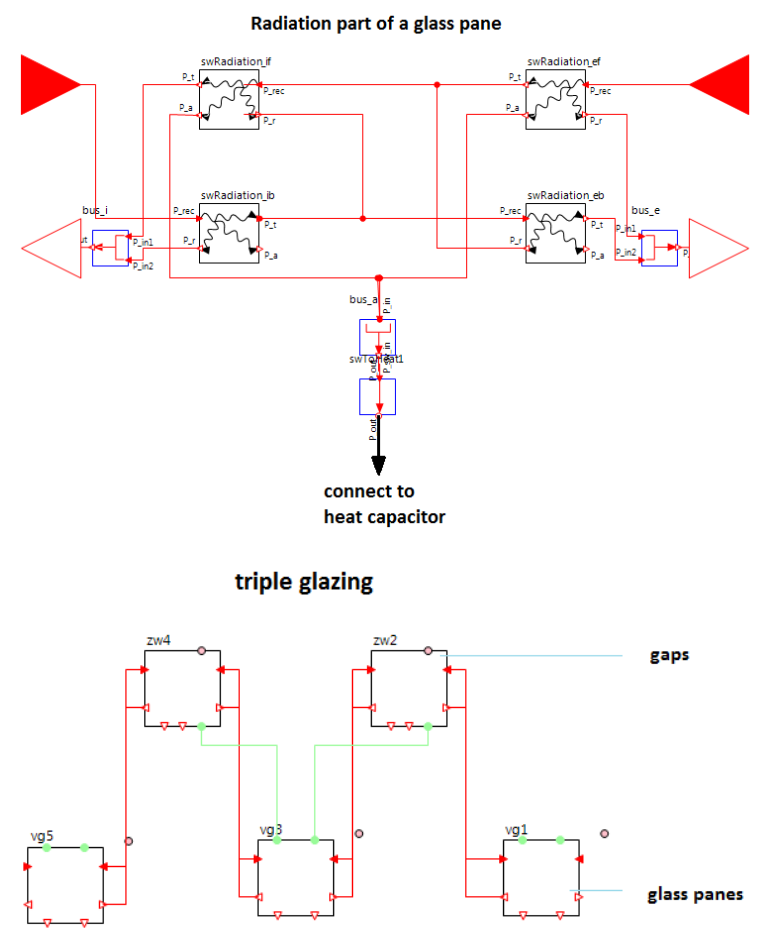

Figure 3. Connections between radiation inside a glass pane and between glass panes and gaps

\subsection{Room-modeling}

Thermal space or room is considered to be the basic unit of building energy performance simulation. Different rooms can be connected by using shared building elements, general boundary conditions and central service systems. Connections inside one room between building elements and other components such as internal loads could be more complicated, and require special attentions. Therefore, a template RoomEmpty is available to help students and engineers build a simulative room model by using "drag-and-drop". In this template, indoor air mass, internal load, radiation distributors and hubconnectors are given. This structure can also provide 
support for automatic generation of a Modelica room model from building information models.

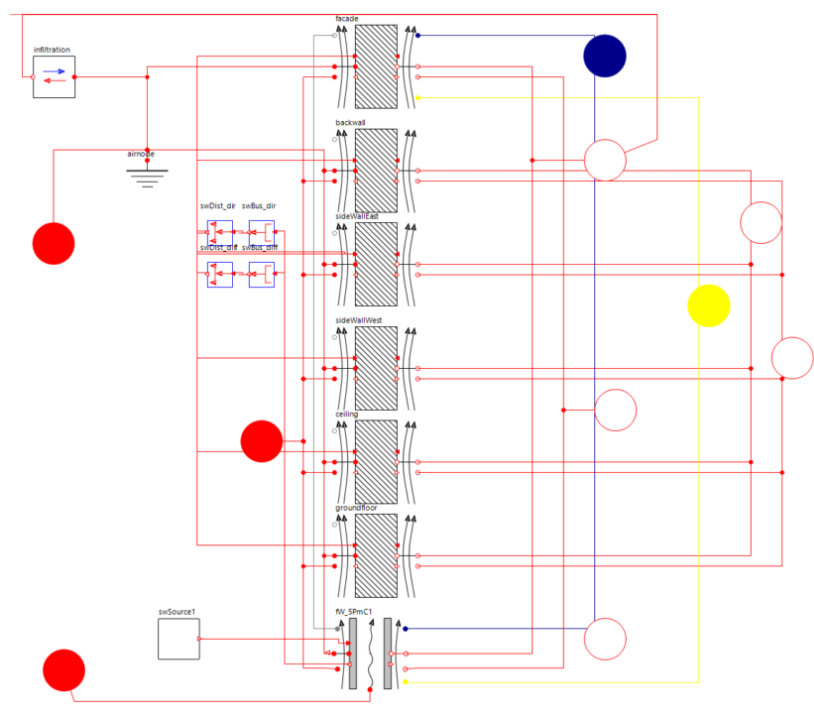

Figure 4. An example room model with six walls (1 of these is an external wall) and a window

\subsection{Heating system}

As part of a master thesis, the existing IBPBuilding library was extended by components for simulation of hot water central heating systems, which are the most important plant types in Germany. The implemented component models are widely accepted best practice models and are primarily taken from existing Modelica libraries, such as the Annex60 library (Wetter and van Treeck, 2016) and the Modelica Buildings Library 2.0 from Lawrence Berkeley National Laboratory (Wetter et al., 2013). The library contains all essential components of pipes, fittings, pumps, heat generators, storages, expansion tanks, transfer systems, control strategies and domestic hot water (DHW) systems to simulate a wide range of complex heating and DHW systems. The newly implemented heating system models of IBPSBuilding library should therefore be seen as a basis for further developments.

\subsection{Compatibility and external connections}

In the framework of a public license, the library is considered to be compatible with the Modelica standard library:

1. The heatports of the sub-package Interface are inherited from the HeatPort of the standard Modelica library. Connectors in the models of IBPSBuilding can be connected directly with standard modules such as with heat sources in Modelica. HeatTransfer package or indirectly through converter-modules.
2. Variables are defined using Modelica.SIunits types.

3. Use of external functions are minimized. Models and functions are verified with test rooms in the environment of OpenModelica and ITI SimulationX.

\subsubsection{Compatibility by encapsulating the parameter sets}

To enhance the compatibility and adaptability of this library, instantiation of room or building models should be conducted automatically by using interfaces or under coordination by platform-software. Automated Modelicacode generation has restrictions such as that no new variables and equations should be constructed, so that the generated graphical representation of models should be readable for engineers. Furthermore, it should be proved that the generated model can be simulated without errors which could be produced by incomplete interpretation and partly combination with manually inserted components.

For this purpose, parameters that essentially can come from an exchangeable data format are encapsulated into records as a property of appropriate building element. The following example shows the instantiated and concretized parameter-set in a concrete wall:

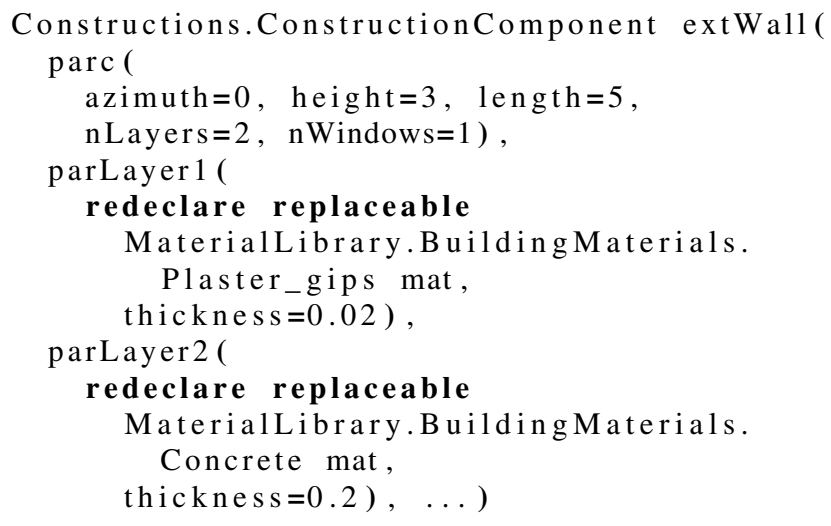

\subsubsection{Weather reader and external Combi Tables}

The IBPSBuilding-package uses CombiTable(e.g. Modelica.Blocks. Tables.CombiTable1D) for indicator-related properties of components (e.g. temperature-related fluid density, wavelengthrelated refractive index or spectral sensitivity) and schedules. An Excel-Macro is developed to convert any data-sheets into Modelica-readable format or time table. A reader of weather data based on the appropriate component in ITI GreenBuilding-package of SimulationX (Unger et al., 2012) is implemented for semantic analysis of CombiTables of weather data according to date and site location, and provides connectors for other components. It is designed to be compatible 
with components of GreenBuilding-package and standard Modelica libraries.

\subsection{Collaboration with students}

Since 2015, for version management the software revision system Git was applied for code organization in teaching activities. Creating modules in different student groups and merging these development trees was the main motivation. In the exercise of the winter semester 2015/16 this idea was realized by creating a Github repository with a basic version of the source code of the IBPSBuilding library and establishing student development groups for the topics convection and longwave radiation processes. This repository is created under the Github-Education program ${ }^{2}$, which is held in private use, and can at the moment only be published with the scope of a virtual 'classroom' with access control of tutors in the lecture. As most of the students with background of architecture and civil engineering had never been working before with software development environments, a lot of technical problems arouse. One of the biggest challenges was to motivate the use of Github, which in the end was only used by the students for downloading the basic version of the library. Pushing new source code versions created by the student groups and merging the development trees had to be done by the supervisors. In the next course Github will be used again, but with more introduction examples to increase the usage of Git as a collaboration platform in and between the groups. Additionally Git will be used in future Master's theses on the library, as in such a thesis more time can be spend on clarifying technical problems. The repository is under GNU GPL license.

\section{Validation}

Thermal building simulation models should be tested to eliminate model errors and to state the quality of the results in relation to calculation accuracy and reality. Quantitative testing can be done with a relative or a physical validation. By a physical validation the simulation model is tested with measured values from a real system, e.g. a well-documented real experiment. In practice, however, a relative validation often is the more convenient choice. In the case of a relative validation a new simulation model is compared to existing simulation models, e.g. by evaluation parameters of national and international validation standards or guidelines. They usually provide different test cases to verify the implemented algorithms and models of certain areas of the program. To evaluate the calculated results comparison values are specified. In this paper, the German guideline

${ }^{2}$ More information: https://education.github.com/.
VDI $2078^{3}$ from the German Association of Engineers (VDI) has been used.

\subsection{Selection of the validation case}

The thermal solver of the IBPS Building library is not based on the 2-capacity (2-K) model by Rouvel and Zimmermann (Rouvel, 2015) as described in the VDI 60071. For simulation programs with another thermal solver than described in VDI 6007-1 and / or radiation model as in VDI 6007-3 the VDI 2078 prescribes a validation according to "Case B"(VDI 2078). This validation case includes:

1. Validation according to test examples 1 to 16 (except 11) of VDI 6020-1 (implicitly included in the test examples of VDI 6007-1 and VDI 2078)

2. Validation of test examples of VDI 6007-1 (Test Example 1-12)

3. Validation of test examples of VDI 2078 (Test Example 1-16)

For this validation case the guideline specifies limiting values for the mean values of the hourly and standard deviation from the reference results of VDI 6020-1. The reference results are based on the $n-K$ model by Rouvel and were calculated using the building simulation program GEBSIMU (Rouvel, 2015). The simulation program results must lie in these limits.

Though, a complete validation of the IBPSBuilding library according to VDI 2078 is not expedient. Part of the test examples of VDI 6020-1 and VDI 2078 require expensive whole-year simulations which clearly complicate the assessment and analysis of test results. Furthermore, features such as window ventilation and daylight calculation, are required in these test examples which are not yet included in the existing library. Therefore, a validation with the test examples of VDI 6007-1 and limiting values according to VDI 2078 is of primary importance for an evaluation of the thermal solver.

\subsection{Type rooms}

For the calculation of the test examples, a simple test room (type room) is defined in the guideline VDI 60071. The construction of the room corresponds, depending on the test example, either to a lightweight construction

\footnotetext{
${ }^{3}$ The guideline VDI 2078 "Calculation of cooling load and room temperatures of rooms and buildings (VDI Cooling Load Code of Practice)" is used to determine the cooling load, the ambient air temperature and the operative room temperature for rooms of all kinds, with and without air conditioning(VDI 2078). The guideline summarizes selected test examples of VDI 6007-1 ("Calculation of transient thermal response of rooms and buildings - Modeling of rooms") and VDI 6020-1 ("Requirements on methods of calculation to thermal and energy simulation of buildings and plants - Buildings"). These should serve both the validation as well as support for programming.
} 
(room type L) or a massive construction (room type $\mathrm{S}$ ). The resulting two different amounts of thermal mass allow to examine the corresponding reactions of the room to these differences. The test room is defined as part of a building and therefore has adiabatic boundaries. From case to case, the outer wall is adjacent to different external climate.

\subsection{Test cases}

The guideline VDI 6007-1 comprises a total of twelve test examples (VDI 6007). The test examples 1 to 7 correspond to the guideline VDI 6020-1 and examine reactions of the room to internal loads and setpoint changes. The test examples 8 to 12 contain important functions of the extended $2-\mathrm{K}$ model by Rouvel, e.g. surface heating or cooling, non-adiabatic internal components, air exchange and several external components.

The thermal reaction of the test room is calculated over a period of 60 days in hourly increments. The predetermined external and internal heat sources and sinks are in temporal pattern of a day the same for the whole period of 60 days. Thus, the transient and the steady state can be compared to the reference results in the guideline.

The kernel of VDI 6007-1 is based on the 2-K model by Rouvel and Zimmermann (Rouvel, 2015). Since the IBPS Building library is designed for the simulation of detailed room models, as suggested by Clarke (Clarke, 2001), the test examples are created accordingly. The basic structure corresponds more to the n-capacity (n-K) model of VDI 6020-1. However, there are some basic differences to the reference $\mathrm{n}-\mathrm{K}$ model of the building simulation program GEBSIMU, as described below.

With the existing components of the IBPS Building library it is not possible to calculate all of the test examples of the VDI 6007-1. It is therefore necessary to implement some additional components or extend existing components that are comprised in the kernel of VDI 6007-1.

\subsection{Component model}

The $\mathrm{n}-\mathrm{K}$ model (of the building simulation program GEBSIMU ) uses a simplified model suggested in VDI 6020-1 for capacitance and resistance of the individual components of a room (VDI 6020). For each single or multilayer component a substitute model is calculated using a chain matrix from concentrated thermal resistances and capacitances. These capacitances and resistances reflect the thermal mass of room components that can be activated. By this, the common solution methods such as Fourier or Laplace transformation can be avoided. The active thermal mass is described by the aperiodic depth of penetration and is calculated regarding the considered period duration.

A substitute model for a wall with any number of different layers can be combined into an equivalent resistor- capacitor ( $\mathrm{RC}$ ) circuit with a maximum of three resistors and two capacitors. Asymmetric thermally loaded components, such as external components or internal components to different tempered neighboring spaces are described by two resistors and one capacitor. Symmetrical thermally loaded components, i.e. adiabatic internal components are modeled as a damped heat storage with one resistor and one capacitor (see Figure 5).
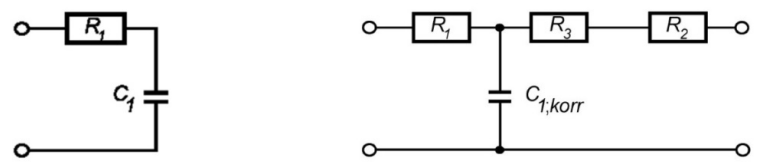

Figure 5. Equivalent circuit for a symmetrical (left) and asymmetrical (right) loaded component (Rouvel, 2015)

The IBPS Building library's equivalent models for single- or multi-layer components are based on the Beuken model. The Beuken model is based on the agreement between the differential equation of the thermal conduction and the processes in an idealized electrical cable (VDI 6020), as shown in Figure 6.

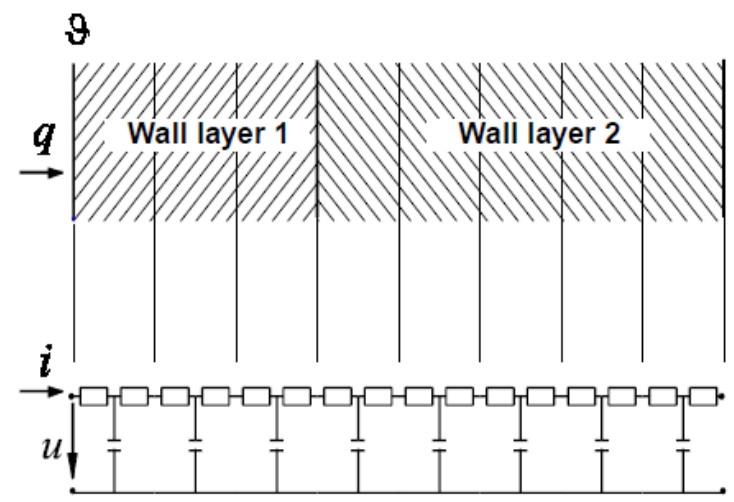

Figure 6. Component as Beuken model (VDI 6020)

In the Beuken model any wall layer is represented by an $\mathrm{RC}$ circuit with two resistors and one capacitor. A component (e.g. a wall) can basically be divided into any number of layers with an equivalent circuit. The degree of subdivision of the wall layers is normally chosen based on the required calculation accuracy but in principal any required calculation accuracy is possible. No requirements are stipulated regarding the boundary conditions (such as linearity etc.). In the IBPS Building library the spatial discretization of component models is limited to one RC circuit per component layer, i.e. per material layer. This allows a significantly faster computation time.

In the IBPSBuilding library based detailed models components to adiabatic boundaries, that is, interior walls, door, floor and ceiling, are approximated by only half of the input thickness. In VDI 6020-1, the "corrected heat storage capacity" of the symmetrically loaded components, i.e. those to adiabatic boundaries, are cal- 
culated in dependence of the aperiodic depth of penetration. The simplified assumption in the detailed model leads to overall smaller heat capacities, whose impacts can be observed in the simulation results. The windows are modeled in accordance with VDI 6020-1 as opaque component with thermal resistance but without heat storage.

\subsection{Longwave radiation}

Longwave radiation exchange between different surfaces in the building simulation program GEBSIMU is assumed to be calculated by the approach for "more complicated" geometrical conditions of the VDI 60201 (VDI 6020). This simplified linear approach assumes that all surfaces of the room are involved in the radiation exchange relative to their size:

$$
\dot{Q}=A \cdot \alpha_{\mathrm{str}} \cdot\left(T_{1}-T_{2}\right)
$$

In VDI 6007-1 the heat transfer coefficient is defined as $\alpha_{\text {str }}=5 \mathrm{~W} /(m K)$ (VDI 6007). According to ISO 6946:2007 "Building components and building elements - Thermal resistance and thermal transmittance - Calculation method" this value corresponds to a temperature of $T_{m}=10^{\circ} \mathrm{C}$. Thus, it is assumed that this value is also used in the GEBSIMU model. The IBPS Building library uses an approach based on the Stefan-Boltzmann law:

$$
\dot{Q}=A \cdot \sigma \cdot \varepsilon \cdot\left(T_{1}^{4}-T_{2}^{4}\right)
$$

This leads to higher radiative heat flows between the walls for temperatures over $10^{\circ} \mathrm{C}$. Therefore, the indoor air temperature on day 60 in the guideline is higher than in our model. This originates in the radiative heat flow from the inner to the outer wall, which is underestimated in the guideline and leads to lower heat losses to the outside.

\subsection{Simulation}

After entering all data the test cases are simulated over the base period of 60 days. The output is given in hourly steps as specified in the directive. By evaluating the results, programming errors in the IBPSBuilding library could be detected and eliminated.

The reference results of validation "Case B" are based on the $\mathrm{n}-\mathrm{K}$ model by Rouvel which is included in VDI 6020-1 (VDI 6020). These were calculated using the building simulation program GEBSIMU version 7.30.0011. The basics of the $n-K$ model were outlined by Rouvel in 1972 based on the detailed Beuken model. The compliance of both models calculation results has been proved by Rouvel (Rouvel, 2015).

\subsection{Results analysis and discussion}

The simulation results of the test cases of VDI 6007-1 are compared to the reference results of validation "Case B" according to the specifications of VDI 2078. The reference results are in this case based on the $n-K$ model as included in VDI 6020-1.

For analysis of the test examples, day 1, 10 and 60 of the base period are compared in respect to room air temperature, operative temperature, and for test examples 6 , 7 and 11, to heating and/or cooling load. By this, initial values, transient response and consistency in steady state can be examined. Figure 7 exemplarily shows the transient response of Test Example 1 over the whole base period of 60 days.

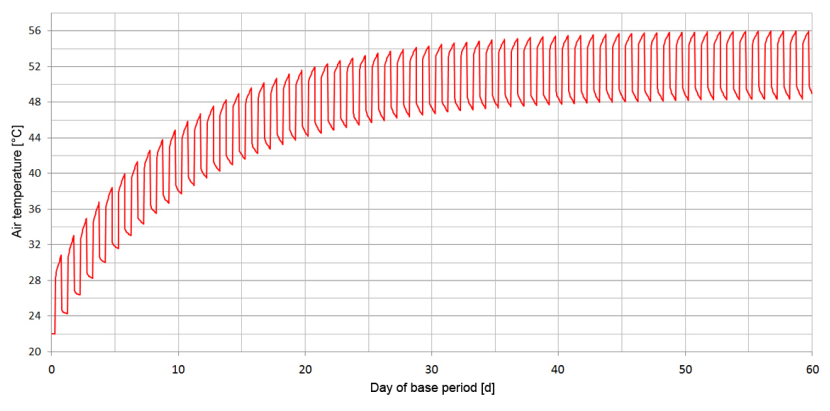

Figure 7. Development of the room air temperature over the simulation period

Figure 8 exemplifies the diurnal variations of the indoor temperatures of Test Example 1. The increase in air temperature due to the internal loads in office hours (6.00 - 18.00 o'clock) can be clearly seen. Compared are the reference results of the 2-K model of VDI 6007-1 and the reference results of the $\mathrm{n}-\mathrm{K}$ model of VDI 6020-1 with the simulation results of the IBPS Building Library. The maximum standard deviation of the hourly variations of the simulation results from the reference results occurs for the room air temperature and the operative temperature on day 1 with $0.28 \mathrm{~K}$. The maximum absolute deviation is about $1.0 \mathrm{~K}$ and occurs on day 60 . The test example shows an overall good compliance with the reference results. However, differences in the calculation engine between the $n-K$ model and the IBPS Building library's model result in significant variations in all test examples.

It is apparent that the indoor air temperature shows a wider fluctuation range on all days than the reference results. This can be justified by the smaller overall effective heat capacity of the zone. As described before, the heat capacity of the reference model is calculated in accordance with VDI 6007-1, i.e. in dependence of the aperiodic depth of penetration. The heat capacity to adiabatic boudaries of the IBPSBuilding library model is simply estimated by half the wall thickness. In this way the total heat capacity of the IBPS Building model is smaller and the room air temperature is responding 
Day 1

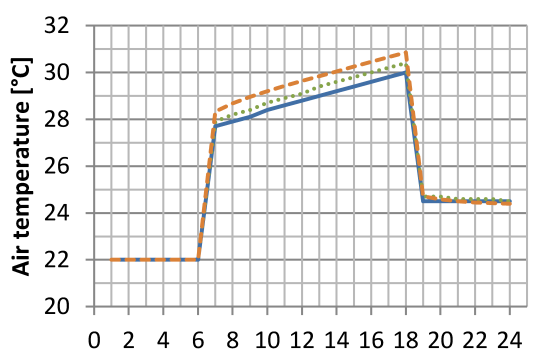

Day 10

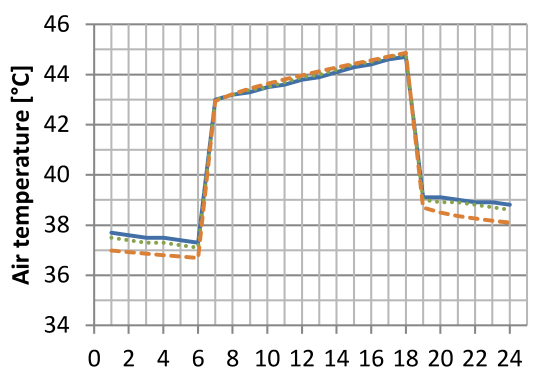

Day 60

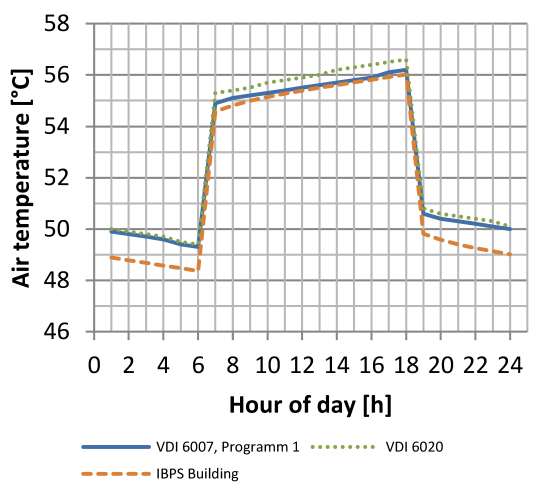

Figure 8. Results of test example 1

faster to heat sources and heat sinks on every day, until it reaches the upper limit in steady state.

Furthermore, the room air temperature of the IBPSBuilding model increases slower during the simulation period than in the reference model. This is attributable to the heat flow from the inner to the outer wall by longwave radiation, which is considered too small in the reference model. As described before, in contrast to the $\mathrm{n}-\mathrm{K}$ model, the IBPSBuilding library does not use the simplified linear approach for the long-wave radiation exchange, but a model based on the Stefan-Boltzmann law. This leads to higher radiative heat flows between the walls at temperatures above $10^{\circ} \mathrm{C}$ and thus to higher thermal losses through the exterior walls. The room air temperature is therefore, on day 60 lower than in the reference results. The two behaviors described above occur in all test examples.

Minor discrepancies at all considered days can be at- tributed to slight differences in the calculation results by using different calculation programs (VDI 6007). Depending on the programming, programming language, compiling, etc. slight differences are inevitable.

In heating and cooling load significantly higher deviations from the reference results are conveyed. In Test Example 11 the room air temperature is in good consistence with the reference results with a maximum standard deviation of $0.47 \mathrm{~K}$ on the 1 st day. Though the system load has high standard deviations from the reference results of $65.6 \mathrm{~W}$ on day 1 and approximately $56.4 \mathrm{~W}$ on day 10 and 60 . The maximum absolute deviation occurs on day 1 with about $190 \mathrm{~W}$.

This is primarily due to the low spatial discretization which aggravates the correct identification of the activated thermal mass. Another factor is the use of a PID (proportional-integral-derivative) controller to determine the required heating and cooling load. Here, the $\mathrm{n}-\mathrm{K}$ model of GEBSIMU according to VDI 60201 uses a discrete analytical approach to determine system loads, which leads to fundamentally different results (VDI 6020).

Evaluation is performed as a statistical analysis by mean value and standard deviation corresponding to VDI 6007-1 (VDI 2078). The mean value is the mean of the hourly variation, i.e. simulation result minus reference result, in the evaluation period. The evaluation period includes day 1, 10 and 60 of the simulation period. The standard deviation is the standard deviation of the hourly variation in the evaluation period. The discrepancies between the program to be validated and the reference results are also compared to the deviations of the 2-K model of VDI 6007-1 to the reference results (see Table $1^{4}$ ).

A successful validation requires the maximum values of the mean values and the standard deviation of the evaluation period to be within the applicable limiting values. The limiting values are $1.0 \mathrm{~K}$ for the mean value and 1.5 $\mathrm{K}$ for the standard deviation of the room air temperature and the operative temperature and $50 \mathrm{~W}$ for the mean value and $60 \mathrm{~W}$ for the standard deviation of the heating or cooling load.

The overall results for room air temperature and operative temperature of the test examples are continuously within the limiting values. However, with heating and cooling load calculation limiting values are not always respected. Although the mean value of the hourly deviation is within the limiting value of $50 \mathrm{~W}$ in all test examples, the standard deviation exceeds the limiting values of $60 \mathrm{~W}$ in Test Example 6 with $60.0 \mathrm{~W}$ and significantly in Test Example 11 with $65.6 \mathrm{~W}$. This can primarily be refered to the low spatial discretization of building components which aggravates the correct identification of the activated thermal mass. Thereby, this problem can be

\footnotetext{
${ }^{4}$ The table includes only the maximum values of the evaluation period. The respective higher values are underlined and are those which apply for validation according to VDI 2078.
} 
easily remedied by expanding the number of RC circuits per component layer of the IBPS Building library's component model.

Table 1. Maximum values of validation results

\begin{tabular}{|c|c|c|c|c|c|c|}
\hline \multirow{4}{*}{$\begin{array}{l}\text { Test Example } \\
\text { mean value } \\
\text { standard deviation }\end{array}$} & \multicolumn{6}{|c|}{ Maximum } \\
\hline & \multicolumn{3}{|c|}{ IBPS Building model } & \multicolumn{3}{|c|}{ VDI 6007-1 model } \\
\hline & Air temp. & Oper. temp. & Sys. load & Air temp. & Op. temp. & Sys. load \\
\hline & $\mathbf{K}$ & K & w & K & $\mathbf{K}$ & w \\
\hline \multirow[t]{2}{*}{1} & $\underline{0,83}$ & $\underline{0,84}$ & & 0,27 & 0,27 & \\
\hline & $\underline{0,28}$ & $\underline{0,28}$ & & 0,16 & 0,15 & \\
\hline \multirow[t]{2}{*}{2} & $\underline{0,42}$ & $\underline{0,42}$ & & 0,34 & 0,35 & \\
\hline & $\underline{0,28}$ & $\underline{0,28}$ & & 0,13 & 0,13 & \\
\hline \multirow[t]{2}{*}{3} & $\underline{0,74}$ & $\underline{0,76}$ & & 0,42 & 0,42 & \\
\hline & 0,50 & 0,49 & & $\underline{1,09}$ & $\underline{1,05}$ & \\
\hline \multirow[t]{2}{*}{4} & 0,61 & 0,60 & & $\underline{0,64}$ & $\underline{0,66}$ & \\
\hline & 0,38 & 0,39 & & $\overline{0,74}$ & $\overline{0,72}$ & \\
\hline \multirow[t]{2}{*}{5} & 0,54 & 0,57 & & $\overline{0,19}$ & $\overline{0,18}$ & \\
\hline & $\overline{0,23}$ & $\overline{0,23}$ & & $\underline{0,26}$ & $\underline{0,25}$ & \\
\hline \multirow[t]{2}{*}{6} & & $\underline{0,09}$ & 23,2 & & 0,04 & 17,1 \\
\hline & & $\overline{0,24}$ & $\overline{60,0}$ & & 0,10 & 40,9 \\
\hline \multirow[t]{2}{*}{7} & $\underline{0,23}$ & 0,17 & $\underline{23,6}$ & 0,17 & $\underline{0,18}$ & 16,0 \\
\hline & 0,24 & $\underline{0,32}$ & 56,0 & 0,18 & 0,17 & 28,7 \\
\hline \multirow[t]{2}{*}{8} & 0,88 & $\underline{0,53}$ & & 0,41 & 0,43 & \\
\hline & 0,73 & 0,33 & & 0,55 & 0,51 & \\
\hline \multirow[t]{2}{*}{9} & $\underline{0,90}$ & $\underline{0,54}$ & & 0,41 & 0,42 & \\
\hline & 0,74 & $\overline{0,33}$ & & 0,55 & 0,53 & \\
\hline \multirow[t]{2}{*}{10} & $\underline{0,44}$ & $\underline{0,41}$ & & 0,16 & 0,17 & \\
\hline & 0,23 & 0,20 & & $\underline{0,26}$ & 0,27 & \\
\hline \multirow[t]{2}{*}{11} & 0,44 & 0,44 & 43,4 & 0,07 & 0,07 & 4,3 \\
\hline & 0,47 & 0,50 & 65,6 & 0,09 & 0,09 & 12,3 \\
\hline \multirow[t]{2}{*}{12} & 0,16 & $\underline{0,17}$ & . & 0,15 & 0,15 & \\
\hline & 0,26 & 0,27 & & 0,25 & 0,26 & \\
\hline
\end{tabular}

\section{Conclusion and outlook}

\subsection{Adoption to IEA EBC Annex 60 frame- work}

As a complement of other Modelica libraries for building energy performance simulation, the development of the IBPSBuilding library is focused on building modeling and simulation with Modelica mainly for educational and teaching purposes. The main structure of this model library and its compatibility is emphasized in this work, so that it can be easily adopted into learning work and used by non-programmers.

Further activities shall link the activities on hand with the ongoing work of the international IEA EBC Annex 60 project. The Annex within the International Energy Agency (IEA) in Buildings and Communities programme (EBC) is a project to promote research and development of new computational methods for energy efficient buildings and communities. The focus is to develop and demonstrate next-generation computational tools for buildings and energy systems within buildings based on Modelica and the Functional Mockup Interface standards (Wetter and van Treeck, 2016).

One of the subtasks of the Annex framework focuses on harmonizing and unifying model development in Modelica. As a result of the Annex work, fragmented developments were merged into a common and open set of Modelica base classes for the various libraries such as the AixLib from RWTH Aachen University, see e.g.
(Remmen et al., 2015), (Fuchs et al., 2015) and the references therein, or the Building library from LBNL Berkeley (Wetter et al., 2013) and others. It is therefore the intention to merge the developments on hand which primarily focus on educational issues with the research- and application-oriented models of the Annex.

\subsection{Validation}

The validation of the IBPSBuilding Library on the basis of VDI Directive 6007-1 with the limit values according to VDI 2078 shows overall good results. Thus demonstrating the resilience of the thermal calculation kernel. By evaluating the results programming errors were detected and remedied, e.g. the incorrect definition of the heat transfer coefficient. In addition, existing components were expanded to include useful additional functions. With the successful validation, the basis for the future development of requested complementary functions has been made, e.g. moisture transport processes, multizone modeling, detailed window models, etc..

\subsection{FLUIDGLASS}

The IBPSBuilding-Package is adopted in the EU-project FLUIDGLASS to support validations of the new façade construction and the integrated control system for fluid and its technical systems. It is a research project coordinated by University of Liechtenstein and funded by European Commission with the seventh Framework Program (EU-FP7 Grant Agreement No. 608509). ${ }^{5}$ In this new façade system in development, two fluidized layers are implemented and proposed to be regulated by an intelligent control system, in order to improve the building energy efficiency by acting as a replacement to cover the functions of shading device, solar thermal collector, heating and cooling elements (Stopper et al., 2013).

In order to accomplish the detailed validation process for the performance of various construction configurations of FLUIDGLASS, an extended version of IBPSBuilding is implemented, which includes:

1. consideration of glass coating and its different optical performances according to direction of irradiation and incident angles;

2. consideration of different behavior with light in different wavelengths;

3. free or enforced convection in a gap between glass panes with detailed calculation method and temperature-related material properties;

4. shading of reveal and its geometric interpretation according to incident solar angles;

\footnotetext{
${ }^{5}$ More information: http://www.fluidglass.eu/
} 
5. visual indicators such as illuminance and luminance;

6. improvement of interactions with 3rd-party libraries, MEP-models and modeling optimization

Additionally, a group of modules are created to help building a detailed FLUIDGLASS model in Modelica, which is compatible with the IBPSBuilding-Package and most of the standard Modelica libraries (mainly SIunits and HeatTransfer). The theoretical numerical parts, especially the behavior according to wavelengths, refer to the former research works and their equation system in the equation solver program EES from F-Chart Software (Gstoehl et al., 2011). The FLUIDGLASS components with IBPSBuilding modules are tested in ITI SimulationX, and are also validated with the EES model and sample measurements.

The extended version of the library is planned to be adopted in network simulations on district level in further steps of FLUIDGLASS project. Approaches of cosimulation are in research. It can collaborate with Matlab and Simulink at the moment in practice by using the COM-interface of SimulationX. Furthermore, the library is adopted in Hardware-in-Loop simulations by interacting with LabVIEW to improve the smart controlling of mechanical systems with FLUIDGLASS. The compatibility of the FLUIDGLASS add-on is also going to be tested with the standard Annex60 Buildings Library.

\section{Acknowledgements}

The authors acknowledge the financial support by the European Commission within the FLUIDGLASSproject (EU-FP7 Grant Agreement No. 608509).

\section{References}

VDI 6020 Blatt 1: Anforderungen an Rechenverfahren zur Gebäude- und Anlagensimulation, 2001-05.

VDI 2078: Berechnung der Kühllast und Raumtemperaturen von Räumen und Gebäuden (VDI-Kühllastregeln), 2012.

VDI 6007 Blatt 1: Berechnung des instationären thermischen Verhaltens von Räumen und Gebäuden - Raummodell, 2012-03.

J Clarke. Energy Simulation in Building Design. Taylor \& Francis, 2001. ISBN 9780750650823. URL http: / / books.google.de/books?id=ksNIQ4kx6UIC.

Marcus Fuchs, Ana Constantin, Moritz Lauster, Peter Remmen, Rita Streblow, and Dirk Müller. Structuring the building performance Modelica model library AixLib for open collaborative development. In Proceedings of 14th IBPSA Conf. Building Simulation 2015, pages 331-338, Hyderabad, 2015.
Daniel Gstoehl, Jochen Stopper, Stefan Bertsch, and Dietrich Schwarz. Fluidised glass façade elements for an active energy transmission control. In World Engineers' Convention, Geneva, 2011.

Shan Hua. Entwicklung einer Schnittstelle zwischen IFCGebäudemodellen und Modelica. 2014.

Peter Remmen, Jun Cao, S. Ebertshäuser, J. Frisch, Moritz Lauster, Tobias Maile, J. O’Donnell, S. Pinheiro, J. Rädler, R. Streblow, M. Thorade, R. Wimmer, Dirk Müller, C. Nytsch-Geusen, and Christoph van Treeck. An open framework for integrated BIM-based building performance simulation using Modelica. In Proceedings of 14th IBPSA Conf. Building Simulation 2015, pages 379-386, Hyderabad, 2015.

Lothar Rouvel. Thermische Gebäudesimulation GEBSIMU - Berechnungsverfahren zum instationären thermischen Gebäudeverhalten, 2015. URL http: / / www - gebsimu . de.

Jochen Stopper, Felix Boeing, and Daniel Gstoehl. Fluid Glass Façade Elements : Influences of dyeable Liquids within the Fluid Glass Façade . In Energy Forum on Solar Building Skins, Bressanone, 2013.

Matthis Thorade, Jörg Rädler, Peter Remmen, Tobias Maile, Reinhard Wimmer, Jun Cao, Moritz Lauster, Christoph Nytsch-Geusen, Dirk Müller, and Christoph van Treeck. An Open Toolchain for Generating Modelica Code from Building Information Models. pages 383-391, sep 2015. doi:10.3384/ecp15118383. URL http://www.ep.liu.se/ecp\{_\}article/ index.en.aspx?issue $=118$; $\operatorname{article}=41$.

René Unger, Torsten Schwan, Beate Mikoleit, Bernard Bäker, Christian Kehrer, and Tobias Rodemann. "Green Building" - Modelling renewable building energy systems and electric mobility concepts using Modelica. Proceedings of the 9th International Modelica Conference, pages 897-906, 2012. doi:10.3384/ecp12076897. URL http://www.ep.liu.se/ecp\{_\}article/ index.en. aspx?issue $=76$; article $=93$.

Christoph van Treeck. Introduction to Building Performance Modeling and Simulation. Habilitation thesis, Technische Universität München, 2010.

Michael Wetter and Christoph van Treeck. IEA EBC Annex 60 Website, 2016. URL http: / / www . iea-annex 60 . org/. Accessed: 2016-04-04.

Michael Wetter, Wangda Zuo, Thierry S Nouidui, and Xiufeng Pang. Modelica Buildings library. Journal of Building Performance Simulation, pages 1-18, mar 2013. ISSN 1940-1493. doi:10.1080/19401493.2013.765506. URL http://dx.doi.org/10.1080/19401493. 2013.765506 . 